\title{
Fabrication and characterization of crystalline copper nanowires by electrochemical deposition inside anodic alumina template
}

\author{
PENG YiTian ${ }^{1,2^{*}} \&$ CHEN QuanFang ${ }^{3}$ \\ ${ }^{1}$ Jiangsu Key Laboratory of Design and Manufacture of Micro/Nano Bio-medical Instruments, School of Mechanical Engineering, Southeast \\ University, Nanjing 211189, China; \\ ${ }^{2}$ Suzhou Research Institute of Southeast University, Suzhou 215123, China; \\ ${ }^{3}$ MEMS and Nanomaterials Laboratory, Mechanical, Materials and Aerospace Engineering Department, University of Central Florida, \\ Orlando, FL 32816, USA
}

Received March 13, 2013; accepted May 15, 2013; published online July 23, 2013

\begin{abstract}
Copper nanowires were fabricated by electrochemical deposition inside anodic alumina template anodized on aluminum substrate. The morphology, composition and structure of the copper nanowires were characterized by means of scanning electron microscopy (SEM), transmission electron microscopy (TEM), high-resolution transmission electron microscopy (HRTEM), energy-dispersive (EDS) and X-ray diffraction spectroscopy (XRD). The results revealed that copper nanowires were dense, continuous, highly-crystalline and uniform with diameters. The electrical properties of copper nanowires wrer characterized with two-terminal electrical measurements. Different current-voltage $(I-V)$ characteristics of single copper nanowire were observed and possible conductive mechanisms were discussed. The crystalline copper nanowires are promising in application of future nanoelectronic devices and circuits.
\end{abstract}

anodic alumina template, electrochemical deposition, copper nanowire, electrical characterization

Citation: Peng Y T, Chen Q F. Fabrication and characterization of crystalline copper nanowires by electrochemical deposition inside anodic alumina template. Chin Sci Bull, 2013, 58: 3409-3414, doi: 10.1007/s11434-013-5965-1

The developments of ever more powerful electronics depend on continued progress in miniaturizing their components. The feature size of integrated circuits has rapidly decreased to nanoscale, due to the improvements in the lithographic materials and processes. Worldwide efforts have been devoted to nanoscale electron transport of one-dimentional nanomaterial both experimentally and theoretically as nanoscale electronic transport could represent the ultimate size of future nanoelectronic devices and circuits. Copper plays an important role in modern electronic circuits in the last decades due to its excellent electrical conductivity and low cost. Currently, copper interconnects are down scaled to the nanometer size. So copper nanowires are attracting considerable interests and expecting to be an essential component of nanoelectronic devices and circuits in the near future $[1,2]$. The electrical properties of copper nanowire behave

\footnotetext{
*Corresponding author (email: pengyitian@gmail.com)
}

different because the mean-free path is a few tens of $\mathrm{nm}$ and the electron wavelength is only a fraction of $\mathrm{nm}$, comparable to the size of an atom [3]. The fabrication of copper nanowires is the prerequisitive to build it into integrated circuits for future application. Numerous techniques have been developed for the preparation of copper nanowires.

Artificial nanopores have emerged as possible mold candidates for building the metal nanowires [4,5]. Copper nanowires were successfully fabricated by templating various types of artificial nanopore material, such as anodic alumina template (AAT) or polymermembranes, mesoporous materials [6-8]. AAT is particularly suited for the fabrication of copper nanowires because it provides a rigid matrix with well-aligned pores, whose diameter and thickness can be easily controlled by changing the anodization conditions. The electrochemical deposition (ECD) inside AAT provides a low-cost, versatile and controllable technique for producing uniform copper nanowires $[9,10]$. The 
extra conductive metal layers were needed in the existed fabrication process of copper nanowires with ECD technique. However the preparation of conductive layer by evaporation or sputtering process is expensive, time-consuming and brings contamination into nanopores [6-9].

Here, copper nanowires were fabricated using ECD technique inside AAT on aluminum substrate. The morphology, composition and structure of the copper nanowires were characterized by means of scanning electron microscopy (SEM), energy-dispersive spectroscopy (EDS), transmission electron microscopy (TEM), high-resolution transmission electron microscopy (HRTEM) and X-ray diffraction (XRD). Electrical properties of copper nanowires are very important for electronic applications as well as understanding the electron transport mechanism at nanoscale. The electrical properties of single copper nanowire were investigated with two-terminal electrical measurement. The possible conductive mechanisms of copper nanowire were discussed based on the different conductive behaviors.

\section{Experimental}

Aluminum plate with thickness of $500 \mu \mathrm{m}$ was used as substrate for the fabrication of copper nanowires. Electropolishing of aluminum plate was conducted in a glass beaker filled with the electropolishing solution. The AAT was prepared via a two-step aluminium anodic oxidation process in $0.3 \mathrm{~mol} / \mathrm{L}$ oxalic acid solution, which was similar to the process described previously $[11,12]$. The schematic drawing of the fabrication of copper nanowires using ECD process inside AAT on aluminum was shown in Figure 1. The detailed process with five steps was described as follows: The first anodization step was conducted under constant voltage of $40 \mathrm{~V}$ in a $0.3 \mathrm{~mol} / \mathrm{L}$ oxalic acid solution, in a thermally isolated electrochemical cell at room temperature for $5 \mathrm{~h}$, during which the electrolyte was stirred under a magnetic stirring system. The alumina layer formed during the first anodization process was removed by immersing in an etching solution of $6 \mathrm{wt} \% \mathrm{H}_{3} \mathrm{PO}_{4}$ and $1.8 \mathrm{wt} \% \mathrm{H}_{2} \mathrm{CrO}_{4}$ at $60^{\circ} \mathrm{C}$ for another $2 \mathrm{~h}$. After etching away the anodized alumina layer, pretextured aluminum structure formed was helpful for forming regular nanopore arrays during the second anodization step. The time and the voltage of first anodization were controlled to determine the regularity and size of the nanopores. The second anodization was carried out under the same conditions as the first step (Figure 1(a)). A subsequent etching treatment was carried out in a $5 \mathrm{wt} \%$ phosphoric acid solution at $30^{\circ} \mathrm{C}$ for $20 \mathrm{~min}$ to remove the barrier layer at the bottom of the AAT (Figure 1(b)). It needs to be noted that the dimension of nanopores of AAT was enlarged during the etching treatment. The length of copper nanowires corresponds to the thickness of AAT which was controlled by the second anodization process of two step anodization process including the time, temperature.

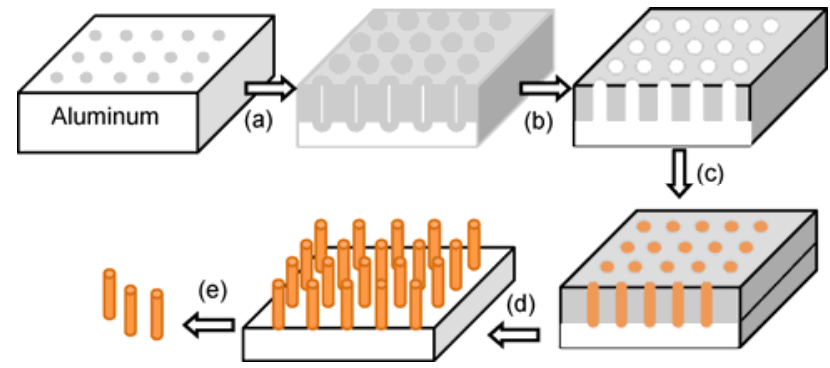

Figure 1 (Color online) Schematic of the fabrication of copper nanowires.

Prior to the ECD experiment, the counter copper electrode (99.999\%) was cleaned by de-ionized (DI) water, acetone and dried in air. Aluminum substrate with AAT was wetted with DI water and then individually immersed in the electrochemical deposition solution consisting of $600 \mathrm{~mL}$ of $0.50 \mathrm{~mol} / \mathrm{L} \mathrm{CuSO}_{4} \cdot 5 \mathrm{H}_{2} \mathrm{O}$ and $0.25 \mathrm{~mol} / \mathrm{L} \mathrm{H}_{3} \mathrm{BO}_{3}(\mathrm{aq})$ for 50 min. Generally, ultrasonic was used to improve wetting of the nanopores prior to electrodeposition [13]. However, this step was eliminated in our process because it may peel off the AAT from aluminum substrate.

The aluminum with AAT as cathode and counter copper electrodes were kept from $1 \mathrm{~cm}$. The ECD experiment was carried out in electrochemical deposition solution by applying a constant voltage of $-0.3 \mathrm{~V}$ for $30 \mathrm{~min}$ at room temperature (Figure 1(c)). The $\mathrm{pH}$ of the electrochemical deposition solution was controlled in the range 3.0 to 3.5 by adding $0.1 \mathrm{~mol} / \mathrm{L} \mathrm{H}_{2} \mathrm{SO}_{4}$ solution. After ECD, the AAT surfaces were polished using $\mathrm{SiC}$ polishing sandpaper to get rid of the excess copper particles sticking to the surface. Then the AAT was rinsed with ethanol and DI water. Then AAT embedded with copper nanowires was immersed into 3 $\mathrm{mol} / \mathrm{L} \mathrm{NaOH}$ solution for $2 \mathrm{~min}$ to dissolve the AAT on aluminum (Figure 1(d)). In the end, the copper nanowires were peeled off from the substrate into DI water solution under ultrasonic treatment (Figure 1(e)).

The morphology and composition of the as-fabricated copper nanowires were characterized with Zeiss ULTRA-55 FEG SEM equipped with Noran System 7 EDS system. A small piece of the AAT embedded with copper nanowires was immersed in $3 \mathrm{~mol} / \mathrm{L} \mathrm{NaOH}$ solution for $10 \mathrm{~min}$ or longer to dissolve away the AAT completely. Then the solution was slowly diluted with DI water and filtered to remove the dissolved AAT and remaining $\mathrm{NaOH}$ solution. Then the copper nanowires were dispersed in methanol solution under ultrasonic treatment. Also a small drop of copper nanowire dispersion was dropped onto copper grids coated with holey carbon film and dried in air for TEM (JEOL 1011) and FEI Technai F30 HRTEM observations. Also the copper nanowires totally disolved in $\mathrm{NaOH}$ were collected by filtration and dried in vacuum. The crystal structure of the copper nanowires was investigated using Rigaku D/Max XRD.

To investigate the electrical properties of copper nano- 
wires, metal electrodes were fabricated to contact with a single copper nanowire. The "find-and-wire" method was described briefly for fabrication of metal-contact single copper nanowire circuits: Firstly, numbered metal grids were written on silicon substrates with a $300 \mathrm{~nm}$ thermally grown $\mathrm{SiO}_{2}$ layer using electron beam lithography (Leica EBPG 5000) and successive deposition of $5 \mathrm{~nm} \mathrm{Cr}$ and 65 $\mathrm{nm} \mathrm{Au}$ as alignment mark. Secondly, the copper nanowires released from the AAT were dispersed in methanol solution under ultrasonic treatments. A droplet of dispersed copper nanowire solution was dropped onto substrate with metal grids. Thirdly, SEM was used to image and locate the deposited copper nanowires refer to grid alignment mark. When the desired single copper nanowire was found, the SEM image was taken for the next step fabrication.

With the SEM images as reference, designed metal contacts with layer of $\mathrm{Cr}(10 \mathrm{~nm}) / \mathrm{Au}(100 \mathrm{~nm})$ were fabricated on the single copper nanowire for making electrical contact using second-time EBL process and successive metal evaporation. Then four gold electrodes were fabricated on the top of the desired single copper nanowire.

The typical SEM image of single copper nanowire-based four contact circuits was shown in Figure 2(a) and the length of the copper nanowire between two electrodes was about $250 \mathrm{~nm}$. The equivalent circuits of single copper nanowire-based four contact circuit were described in Figure 2(b). Two terminal copper nanowire-based circuits were also fabricated using the same method for investigation of

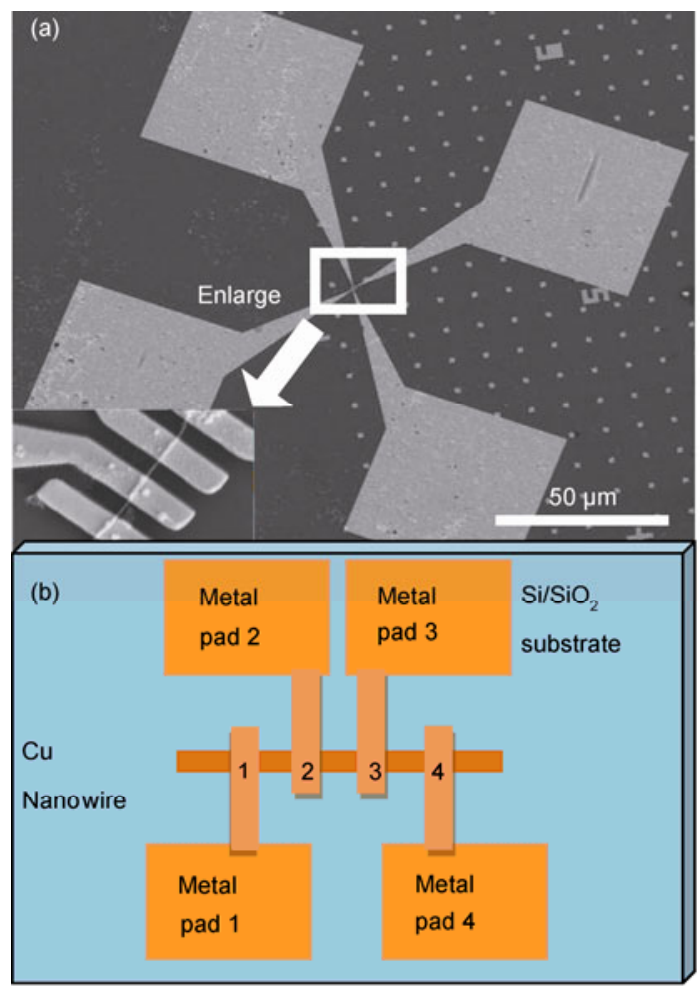

Figure 2 (Color online) SEM image (a) and schematic diagram (b) of the copper nanowire-based four contact circuits. electrical properties.

In order to investigate the conductive behavior of copper nanowires, electrical measurements were performed using straightforward two-terminal method by applying a step voltage to the single copper nanowire and recording the current with $\mathrm{pA}$ resolution using a semiconductor parameter analyzer (Agilent technologies, 4155C Model) coupled with a probe station.

\section{Results and discussion}

A typical SEM image of the copper nanowire array is shown in Figure 3 after the complete removal of AAT. Figure 3(a) and (b) show the top and cross-section view of copper nanowire array and reveal that large quantities of copper nanowires with high packing density had been deposited in the form of wires inside the nanochannels of the AAT. Figure 3(c) and (d) show the SEM and EDS characterization of copper nanowires deposited on silicon substrate. Two evident copper and silicon peaks were observed in the EDS spectrum. Silicon peak comes from silicon substrate and copper peak comes from copper nanowire.

The diameters of the copper nanowires range from 50 to $60 \mathrm{~nm}$, with an average of $55 \mathrm{~nm}$, correspongding with the nanochannel diameters of the AAT. It is important to point out that the diameters and lengths of copper nanowires were controlled by the second anodization process of two step anodization processes.

Figure 4(a) show that the copper nanowires are dense, continuous and relatively uniform in diameter throughout the entire length of the nanowires. This typical SAED pattern of a single copper nanowire (see the insert of Figure 4(a)) shows several continue rings and some diffraction

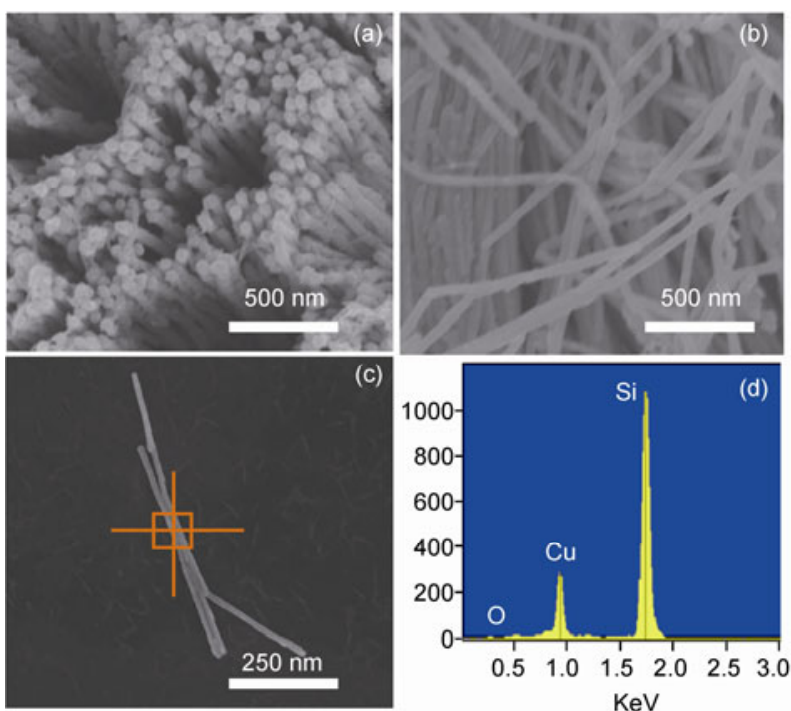

Figure 3 SEM images of copper nanowire array after removing the AAT. (a) Top view, (b) cross-section view, (c) two copper nanowires on silicon, and (d) EDS of copper nanowires on silicon. 

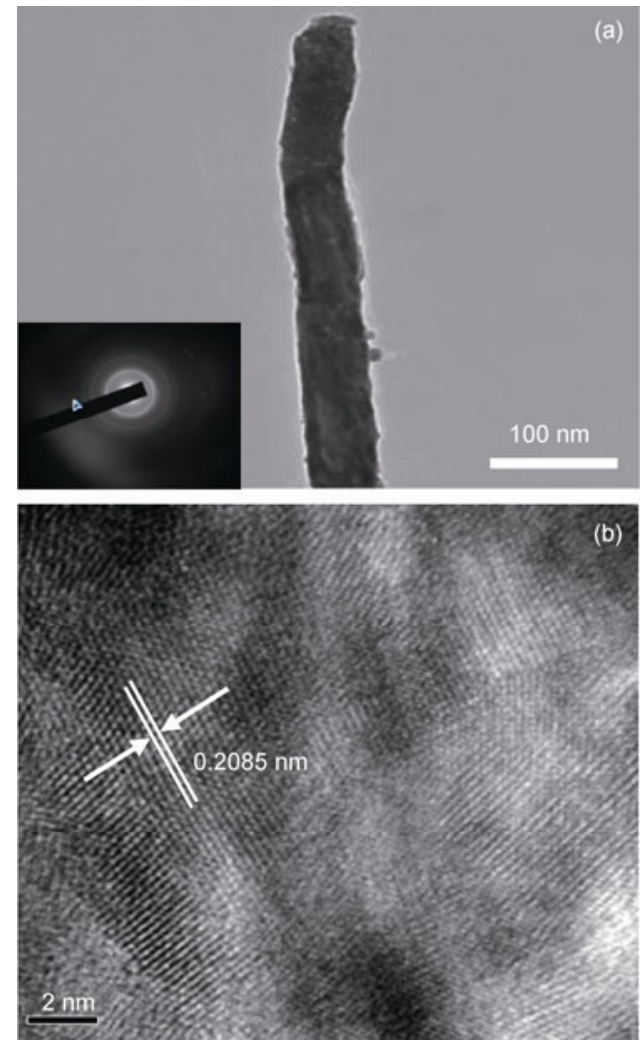

Figure 4 TEM (a) and HRTEM (b) image of copper nanowire.

spots, indicating that the copper nanowires are highly crystalline and have a polycrystalline structure. The diameter distribution of the copper nanowires obtained from TEM images agrees well with the SEM characterization.

A typical HRTEM image of single copper nanowire in Figure 4(b) reveals that most area of copper nanowire has the (111) lattice fringes with lattice spacing around 0.2085 $\mathrm{nm}$. It was noted that nanocrystalline area occurred and hence the copper nanowires were high crystalline structure along preferred plane (111) direction.

Three peaks can be seen in the XRD spectra (Figure 5) of

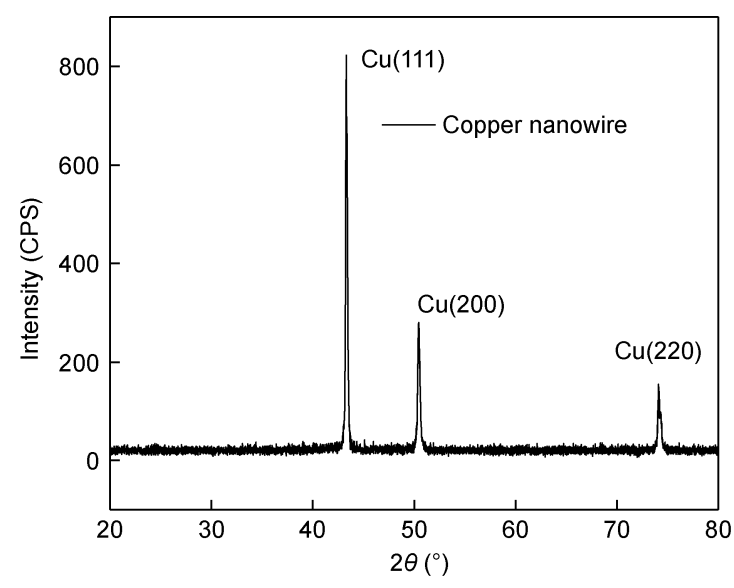

Figure 5 XRD spectrum of the copper nanowires. the copper nanowires, the highest peaks at $2 \theta=44.2^{\circ}$ corresponding to (111) crystal plane and the other two lower peaks at $52.4^{\circ}$ and $73.9^{\circ}$ corresponding to the crystal plane (200) and (220), respectively. All the XRD peaks and intensities indicate that copper nanowires have a polycrystalline structure and a preferential growth direction along the (111) crystal plane, which was consistent with the observation of HRTEM.

Figure 6 shows the typical $I-V$ curves of single copper nanowire with a diameter about $55 \mathrm{~nm}$ between any two electrodes (from electrode 1 to electrode 4 in Figure 2(b)) at room temperature. The scanning voltage was from -1 to 1 $\mathrm{V}$. The nonlinearity and nonsymmetric $I-V$ characteristics can be seen in Figure 6. The nonlinear phenomena may come from the copper oxide layer subject to the exposure to aerial oxygen when single copper nanowire was transformed and placed under electrodes. Moreover, the extremely high surface to volume ratio of the single copper nanowire makes it quite sensitive to oxidation. Both $\mathrm{Cu}(\mathrm{I})$ and $\mathrm{Cu}(\mathrm{II})$ oxides are semiconductors with wide band gap. Two schottky diodes between copper oxide and gold electrodes in series formed a double diode like $I-V$ characteristic curve. The resistivity of the copper nanowires was calculated using a universal four contact method [14] which eliminated the contact resistance between copper nanowire and electrodes. Resistivity of five copper nanowires fabricated in the same batch were measured and calculated using four contact method.

The calculated resistivity of single copper nanowire was around $2.38 \times 10^{-2} \Omega \mathrm{m}$. The resisitivity of copper nanowire is far larger than bulk copper. The high resistivity could attribute to the electron surface scattering and oxidation of copper. Single copper nanowire has a very large aspect ratio, the electrons experience numerous collisions with the single copper nanowire boundary during conduction. Then the mean free path of electrons in single copper nanowire is shorter than in bulk copper $(45 \mathrm{~nm})$. Another possibility for such a phenomenon is presence of a potential barrier between the single copper nanowire and gold pad because

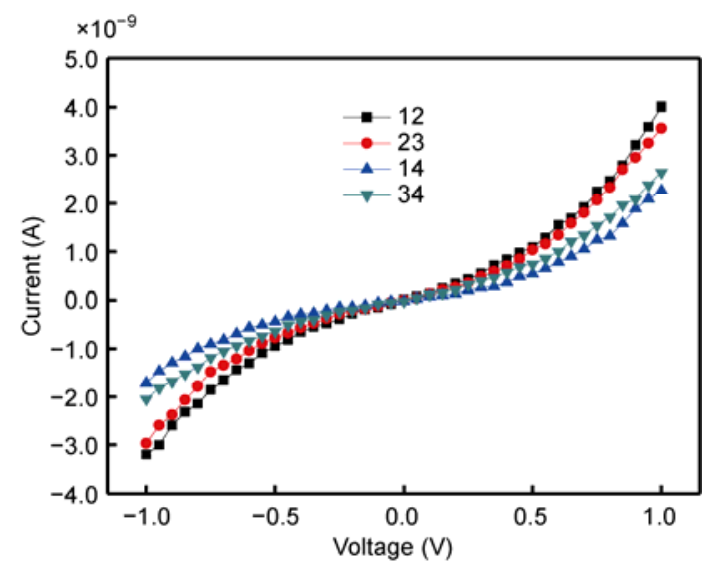

Figure $6 I-V$ curves of the single copper nanowire-based four-contact circuits. 
oxidation. The nonlinear $I-V$ characteristics of copper nanowire could be applicable as the basis of functional nanoelectronic devices.

Figure 7 shows another typical $I-V$ curve of single copper nanowire with the diameters of about $55 \mathrm{~nm}$ while the voltage ranges from -1 to $1 \mathrm{~V}$. At the beginning, the $I-V$ curve obviously demonstrates a linear $I-V$ behavior, indicating a metallic behavior of the single copper nanowires and the electrical contact is almost ohmic. Then the range of scanning voltage was enlarged from -10 to $10 \mathrm{~V}$ with a step width of $0.5 \mathrm{~V}$. It can be observed that the electrical properties of single copper nanowires do not follow ohmic law from -10 to $10 \mathrm{~V}$, while it was obviously nonlinear.

When the biasing voltage is lower than $5 \mathrm{~V}$, there exists a slight deviation from the linearity. This probably arises from the nonideal contacts between $\mathrm{Au}$ electrodes and the single copper nanowire. The barriers exist for the mismatch of the work function between the copper nanowire and $\mathrm{Au}$ electrodes. The carrier density is not large enough to neglect the barriers at room temperature. The curve becomes more linear with the biasing volatge is larger than $5 \mathrm{~V}$. The current values respond to the variation of the potential bias (from -10 to $10 \mathrm{~V}$ ). The maximal current of the single copper nanowire is $70.7 \mathrm{nA}$ when the biasing voltage is equal to $10 \mathrm{~V}$, while the minimal current is $-35 \mathrm{nA}$ when the biasing voltage is at $-10 \mathrm{~V}$. There is a sharp current incremen at
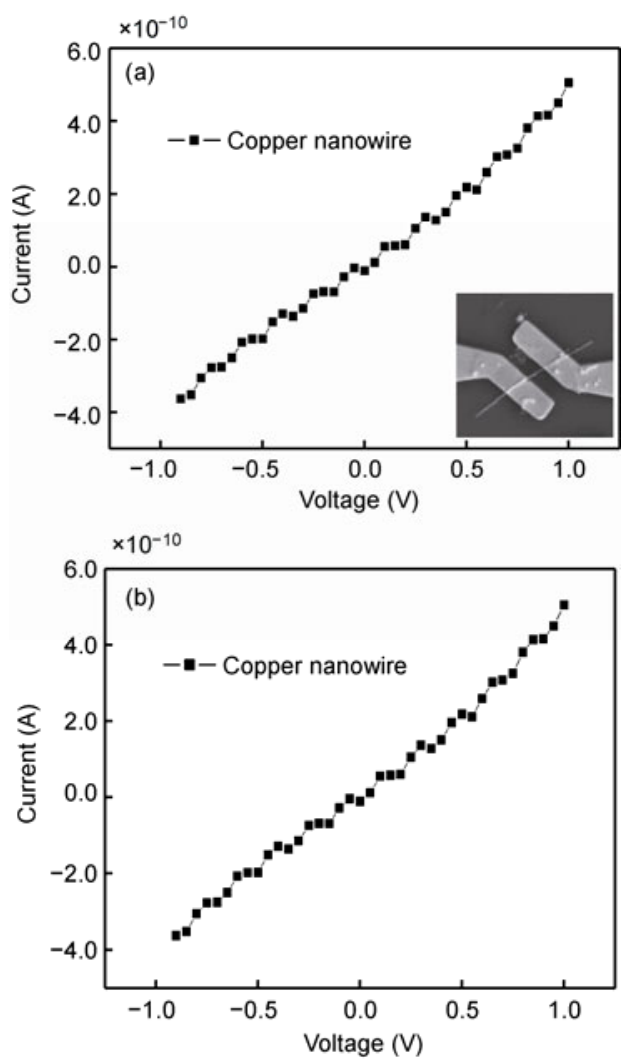

Figure $7 \quad I-V$ curve of the copper nanowire-based two contact circuits at voltage from -1 to $1 \mathrm{~V}$ (a) and -10 to $10 \mathrm{~V}$ (b). Inset was SEM image of two-probe copper nanowire circuits. about $5 \mathrm{~V}$ and the $I-V$ curve gets more linear denotes the influence of barriers gets less above $5 \mathrm{~V}$ as seen in Figure 7. Different barriers between the single copper nanowires and gold electrodes could be reason of the unsymmetric electrical properties [15].

Such a phenomenon was generated from a tunnelling barrier at the copper nanowire-gold junction whose effect gradually collapses as a function of increasing biasing voltage [16]. So conduction electrons possess enough mobility to overcome the energy barrier and the influence of the barrier on the transport was decreased. As more electrons pass through the barriers, the widths of the barriers reduce. The potential barriers gradually collapse as the increase of biasing voltage barriers. The surface condition, crystal structure and quality, chemical composition, crystallographic orientation are also important parameters influence the electron transport of copper nanowires $[17,18]$.

\section{Summary and conclusions}

Copper nanowires were fabricated by ECD of metal copper inside the nanochannels of AAT on aluminum. The individual copper nanowire was high-crystalline, dense, continuous and uniform in diameter throughout the entire length. The simple and inexpensive technique could be extended to fabricate other kinds of metal nanowires without metal evaporation process. Different electrical characteristics of single copper nanowire provides more opportunities with excellent performance application in the nanoelectronic devices and circuits. Further studies on the conductive mechanism of copper nanowire will be performed soon.

This work was supported by the National Natural Science Foundation of China (51002030), International Cooperation Project in Suzhou (SH201117), Excellent Young Teacher Support Plan of Southeast University, in part by the New Century Talents of Ministry of Education (NCET-11-0095), and the PhD Program Foundation of Ministry of Education of China (20100092120006).

1 Gao T, Meng G W, Wang Y W. Electrochemical synthesis of copper nanowires. J Phys: Condensed Matt, 2002, 14: 355-363

2 Riveros G, Gomez H, Cortes R M. Crystallographically-oriented single-crystalline copper nanowire arrays electrochemically grown into nanoporous anodic alumina templates. Appl Phys A, 2005, 81: 17-24

3 Molares M E T, Hohberger E M, Schaeflein C, et al. Electrical characterization of electrochemically grown single copper nanowires. Appl Phys Lett, 2003, 82: 2139-2142

$4 \mathrm{Xu} \mathrm{H}$, Zhang H C, Jiang L. Building bio-inspired artificial functional nanochannels: From symmetric to asymmetric modification. Angew Chem Int Ed, 2012, 51: 5296-5307

5 Gao J, Guo W, Geng H, et al. Layer-by-layer removal of insulating few-layer mica flakes for asymmetric ultra-thin nanopore fabrication. Nano Res, 2012, 5: 99-108

6 Green S, Cortes A, Riveros G, et al. Optical properties of copper and silver nanowires grown in a nanoporous alumina template. Phys Status Solidi C, 2007, 4: 340-343

7 Pang Y T, Meng G W, Zhang Y, et al. Copper nanowire arrays for infrared polarizer. Appl Phys A, 2003, 76: 533-536

8 Cao H Q, Wang L D, Qiu Y, et al. Synthesis and $I-V$ properties of 
aligned copper nanowires. Nanotechnology, 2006, 17: 1736-1739

9 Wang X J, Song W, Li Z S. Fabrication of superhydrophobic AAOAg multilayer mimicking dragonfly wings. Chin Sci Bull, 2012, 57: 4635-4640

10 Gelves G A, Murakami Z T M, Krantz M J, et al. Multigram synthesis of copper nanowires using ac electrodeposition into porous aluminium oxide templates. J Mater Chem, 2006, 16: 3075-3083

11 Kokonou M, Nassiopoulou A G, Giannakopoulos K P. Ultra-thin porous anodic alumina films with self-ordered cylindrical vertical pores on a p-type silicon substrate. Nanotechnology, 2005, 16: 103-106

12 Masud H, Yamad H, Sato M. Highly ordered nanochannel-array architecture in anodic alumina. Appl Phys Lett, 1997, 71: 2770-2772

13 Li A P, Muller F, Birner A, et al. Fabrication and microstructuring of hexagonally ordered two-dimensional nanopore arrays in anodic alumina. Adv Mater, 1999, 11: 483-488

14 Gu W H, Choi H G, Kim K K. Universal approach to accurate resistivity measurement for a single nanowire: Theory and application.
Appl Phys Lett, 2006, 89: 253102-253104

15 Li L, Yang Y W, Fang X S, et al. Diameter-dependent electrical transport properties of bismuth nanowire arrays. Solid State Commun, 2007, 141: 492-496

16 Shao P R, Deng S Z, Chen J. Fabrication and field emission performance of arrays of vacuum microdiodes containing $\mathrm{CuO}$ nanowire emitters grown directly on glass without a catalyst. Chin Sci Bull, 2011, 56: 906-911

17 Mohl M, Pusztai P, Kukovecz A, et al. Low-temperature large-scale synthesis and electrical testing of ultralong copper nanowires. Langmuir, 2010, 26: 6496-6502

18 Zhou X, Dayeh S A, Aplin D, et al. Scanned electrical probe characterization of carrier transport behavior in InAs nanowires. J Vac Sci Technol B, 2006, 24: 2036-2041

19 Xu L P, Yuan Z H, Zhang X G. Fabrication of multi-level branched metal nanowires by AAO template electrodeposition. Chin Sci Bull, 2011, 56: 2055-2058

Open Access This article is distributed under the terms of the Creative Commons Attribution License which permits any use, distribution, and reproduction in any medium, provided the original author(s) and source are credited. 